\title{
Parameter choice for optimized digital image correlation
}

\author{
Karen Triconnet ${ }^{\mathrm{a}}$, Katell Derrien ${ }^{\mathrm{a}}$, François Hild ${ }^{\mathrm{b}}$, \\ Didier Baptiste ${ }^{\mathrm{a}, *}$ \\ a Laboratoire d'Ingénierie des Matériaux (LIM), \\ ENSAM de Paris/CNRS-UMR 8006, \\ 151 Boulevard de l'hôpital,75013 Paris,France. \\ Email: Karen.Triconnet@paris.ensam.fr, Katell.Derrien@paris.ensam.fr, \\ Didier.Baptiste@paris.ensam.fr \\ ${ }^{\mathrm{b}}$ Laboratoire de Mécanique et Technologie (LMT-Cachan), \\ ENS de Cachan/CNRS-UMR 8535/Université Paris 6, \\ 61 avenue du Président Wilson,94235 Cachan Cedex,France. \\ Email: Francois.Hild@lmt.ens-cachan.fr
}

\begin{abstract}
Digital image correlation is a photomechanical technique utilized to measure displacement fields. Although the method has been used successfully in a variety of applications, there is a need for guidelines to choose correlation parameters and for an understanding of their link with the analyzed texture and their effect on the uncertainty of measured quantities. In this paper, two criteria are proposed from a texture study to evaluate the best compromise between measurement uncertainty and spatial resolution prior to any mechanical test. These criteria are then validated on a priori uncertainty evaluations with different textures, and on a real experiment when comparing image correlation results with strain gauge data.
\end{abstract}

Preprint submitted to Elsevier Preprint 23 October 2008 
Key words: digital image correlation, texture, optimization

\section{Introduction}

The measurement of $2 \mathrm{D}$ or $3 \mathrm{D}$ surface motions in an image sequence can be performed by pattern matching of various "markers" [1]. An image is represented by a gray level matrix. During a mechanical test, the intensity map is advected by a displacement field to be determined. The measurement of the displacement is an ill-posed problem [2] since it can only be determined along the direction of the intensity gradient. This shows that the property of the underlying texture influences the performance of the measurement technique. One of the most popular matching procedure aims at maximizing the cross-correlation function $[3,4]$ by seeking, for example, piece-wise constant displacements in interrogation windows.

Two types of situations arise in practice. First, the natural texture is sufficient to use a correlation algorithm. For example, the deformation of mineral wool samples can be analyzed with no special preparation [5]. Similarly, the roughness of a ceramic sample can be sufficient to study displacement fields in a notched beam [6]. Alternatively, artificial markers can be deposited on the surface of interest to create a random texture, e.g., a black and white layer can be sprayed [7]. In both situations, there is a need for the characterization the "quality" of the texture when correlation techniques are to be used and one wants to assess the displacement and/or strain uncertainties.

To evaluate the performance of a correlation algorithm, purely artificial pat-

* Corresponding author. Tel: +33140442016. 
terns can be simulated. For instance, Sutton et al. [8] use a decreasing exponential function as a model for the intensity pattern. The generation of black dots on a white background can also be described by using a Poisson-point process and the picture is the result of the union of all the generated dots [9]. Characteristic properties of this type of texture are easy to derive in terms of average intensity and two-point correlation properties $[9,10]$. For speckles, statistical models have also been proposed to characterize the pattern observed from the diffraction phenomenon [11-15]. The advantage of these texture models is that they can be used to evaluate precisely the performance of a correlation algorithm since the displacement can be applied to each pattern with numerical accuracy. However, the previous models are idealizations of practical textures that can take very different patterns.

When performing experiments, the random texture has not always the desired features and the user may wonder whether the correlation technique will yield robust results. An a priori analysis of the texture is desirable before performing the actual experiment. Under these circumstances, a texture analysis is helpful and artificial motions can only be applied artificially by gray level interpolation (e.g., linear, spline [8]) or by Fourier transform by using the shift-modulation property [16]. An alternative route consists in acquiring a set of pictures of the same surface and computing the corresponding uncertainties. This procedure characterizes the resolution of the technique [17] and does not necessarily yield the largest sub-pixel uncertainty levels as will be shown.

In this paper, three different ways of analyzing a texture are proposed when a correlation technique is used to determine $2 \mathrm{D}$ displacement fields. The present goal is to estimate the sub-pixel displacement errors of a correlation code, and 
the effect on strain uncertainties. Section 2 recalls the basics of the digital image correlation algorithm used herein, and introduces the method used to analyze the texture. Then, two criteria are established (Section 3), tested on thirty one different textures and validated on an experimental application with strain gauge measurements to compare results (Section 4). An alternative approach is proposed leaving more freedom to the user, which is also more time consuming.

\section{Displacement measurement by image correlation: The role of the texture}

To determine a displacement field, one uses two pictures (one corresponding to the reference state, and the other one of the deformed state of a surface). One considers a set of windows in the reference picture (i.e., a square region of $N \times N$ pixels) that will be referred to as Zone Of Interest (ZOI), and their corresponding positions are sought in the "deformed" picture. In the following, it is assumed that the separation between neighboring ZOI centers is constant and equal to $\Delta P$ (this parameter characterizes the "measurement mesh").

\subsection{Correlation procedure}

The displacement of a ZOI in the deformed picture with respect to that in the reference picture is a two-dimensional shift of an intensity signal digitized by, say, a CCD or CMOS camera. To estimate a shift between two signals, one of the standard approach utilizes a correlation function. One considers signals $g(\boldsymbol{x})$ that are merely perturbations of a shifted copy $f(\boldsymbol{x}-\boldsymbol{u})$ of some 
reference signal $f(\boldsymbol{x})$

$$
g(\boldsymbol{x})=f(\boldsymbol{x}-\boldsymbol{u})+b(\boldsymbol{x})
$$

where $\boldsymbol{u}$ is an unknown displacement (here assumed to be uniform over the ZOI) and $b(\boldsymbol{x})$ a random noise. To evaluate $\boldsymbol{u}$, one minimizes the norm of the difference between $f(\boldsymbol{x}-\boldsymbol{U})$ and $g(\boldsymbol{x})$ with respect to a trial displacement $\boldsymbol{U}$

$$
\boldsymbol{U} \min \|g-f(.-\boldsymbol{U})\|^{2}
$$

If one chooses the usual quadratic norm $\|f\|^{2}=\int f^{2}(\boldsymbol{x}) \mathrm{d} \boldsymbol{x}$, the previous minimization problem is equivalent to maximizing the quantity $h(\boldsymbol{U})$

$$
h(\boldsymbol{U})=(g \star f)(\boldsymbol{U})=\int g(\boldsymbol{x}) f(\boldsymbol{x}-\boldsymbol{U}) \mathrm{d} \boldsymbol{x}
$$

where $\star$ denotes the cross-correlation product. Furthermore, when $b$ is a white noise, the previous estimate is optimal. The computation of a cross-correlation is performed either in the original space, or in Fourier space, by using a fast Fourier transform (FFT)

$$
g \star f=\operatorname{NFFT}^{-1}(\mathrm{FFT}[g] \cdot \overline{\mathrm{FFT}[f]})
$$

where the complex conjugate is overlined, and $N^{2}$ is the number of samples in the Fourier transform (to use "fast" algorithms [18], it is required that $N=2^{n}$ pixels, where $n$ is an integer). This condition may be relaxed by using zero-padding or neutral padding techniques [19]. In the sequel, it will be assumed that the ZOI size $\ell$ is characterized by the integer $\ell=2^{n}$. 


\subsection{Ultimate performance}

Let us evaluate the sensitivity vector of a correlation technique. As a first approximation, small displacements $\boldsymbol{U}$ are sought, and one can consider a first order Taylor expansion of $g$ in Equation (1) with respect to $\boldsymbol{U}$

$$
g(\boldsymbol{x}) \approx f(\boldsymbol{x})-\boldsymbol{U} \cdot \boldsymbol{\nabla} f(\boldsymbol{x})
$$

which shows that the sensitivity vector of digital correlation is $\nabla f$ and that the displacement evaluation is a priori an ill-posed problem since only displacements along the gradient vector can be detected. It is partially solved by a regularization over small zones, e.g.,the ZOIs defined earlier, themselves defining the spatial resolution for a displacement measurement (i.e., the minimum distance to have independent measurements). Furthermore, contrary to other optical techniques such as moiré interferometry, the sensitivity varies from zone to zone since it is directly related to the texture of the region of interest. This result explains why a texture analysis is useful to evaluate actual performances of a digital image correlation software. The sensitivity $S(\boldsymbol{n})$ associated to each displacement direction $\boldsymbol{n}$ is given by

$$
S(\boldsymbol{n})=\frac{1}{\boldsymbol{n} \cdot \boldsymbol{\nabla} f}
$$

and its maximum value is denoted by $S_{\max }$. Equation (5) also shows that the smallest measurable displacement is obtained for the smallest picture difference (i.e., one gray level) and the highest sensitivity. It scales as

$$
U_{\min } \propto S_{\max }
$$


where $S_{\max }$ is the inverse of the maximum picture gradient. This value has to be corrected for to account for noise associated to the image formation and acquisition process as well as the fact that the CCD sensor does not cover the whole pixel surface. It is expected that the actual estimate is greater than the theoretical value. In practice, $1 / S_{\max }$ is only a fraction of the dynamic range and not all ZOIs achieve this value. Consequently, the practical discrimination threshold is a quantity that strongly depends on the texture, in particular its standard deviation. By considering a uniform distribution of gray levels ranging from 0 to $G_{\max }$, the maximum gradient is related to the standard deviation $\sigma$ by

$$
S_{\max }=\frac{1}{G_{\max }}=\frac{1}{\sqrt{12} \sigma}
$$

so that, for the sake of simplicity, the standard deviation of the gray level distribution will be considered in the texture analysis.

To evaluate sub-pixel displacements, the cross-correlation function is interpolated. For instance, bi-parabolic interpolation can be used and a one hundredth pixel standard uncertainty $[17]$ is achieved $[8,16]$ for 8 -bit pictures. To be consistent with the present approach, the interpolation and correlation product will be considered in the next section to introduce texture criteria to determine the performance in terms of displacements (and strains) measured by image correlation.

\section{Texture analysis}

In practice, a very large variety of pictures is handled when using image correlation techniques. The image "quality" is one key parameter for evaluating 
the performances of a digital image correlation (DIC) technique. Yet, other experimental parameters influence the quality of a picture such as:

- performances of CCD or CMOS sensors (e.g., digitization, read-out noise, black current noise, photon noise [20]);

- position and intensity of the light source with respect to the specimen and the camera.

The image digitization still uses mostly 8-bit encoding sensors. However, CCD cameras digitizing with 10, 12 or even 16 bits are now available. When using an 8-bit CCD camera, a pixel can take any value of the $2^{8}$ dynamic range. Under a uniform distribution hypothesis, a lower bound to the discrimination threshold $u_{\min }$ is given by (see Equation (7))

$$
U_{\min }=\frac{1}{\Delta N_{g}}=\frac{1}{\sigma \sqrt{12}}
$$

where $\Delta N_{g}$ is the signal range, here equal to the dynamic range $2^{8}$ and $\sigma$ the standard deviation associated with a uniform distribution. For an 8-bit digitization, a correlation algorithm will not detect displacements less than $2^{-8}$ pixel. An estimate of the discrimination threshold for strains $\epsilon_{\min }$ consists in considering the minimum displacement variation equal to the discrimination threshold over a gauge length equal to $2 \Delta P$

$$
\epsilon_{\min }=\frac{U_{\min }}{2 \Delta P}
$$

since a displacement gradient is usually computed by either using a centered difference scheme or enhanced algorithms [21,22], some of them also using a neighborhood of 8 points in addition to the considered one. For a separation of 32 pixels, and an 8-bit digitization, a lower bound to the discrimination 
threshold is of the order of $6 \times 10^{-5}$. These ultimate performances may be far from actual performances due to the quality of the texture. There is therefore a need for criteria to help the user choose the best possible correlation parameters, namely, the ZOI characterized by $n$, and $\Delta P$.

To obtain the maximum number of independent displacement measurements, the user wants to determine the lowest possible ZOI size (i.e., the spatial resolution of a DIC technique when displacements are sought) associated to the experiment that is performed. For a displacement measurement, as illustrated in Figure 1 by any open circle, the spatial resolution corresponds to the ZOI size, and independently of the separation $\Delta P$. On the left-part of Figure 1, the separation is equal to the ZOI size whereas on the right-part the separation represents half the size of the ZOI. By applying an enhanced algorithm [22], Figure 1 shows that the data used for a strain measurement (i.e., solid circles) are located in the dashed square area. The corresponding spatial resolution associated to strain measurements is defined ${ }^{1}$ as $\sqrt{\ell^{2}+(2 \Delta P)^{2}}$ pixels. Figure 1 illustrates two different cases where the spatial resolution for displacement measurement is equal to $\ell$ but on the left-part the strain spatial resolution is equal to $\sqrt{5} \ell$ and on the right-part to $\sqrt{2} \ell$ due to the choice of different separations. However, in the last case, the displacement measurements are not independent.

$\overline{1}$ the evaluation of a strain can be considered as the successive application of two windows, the first one of size $\ell$ to determine the displacement data, and a second one of size $2 \Delta P$ in the present case for the derivation stage 


\subsection{A priori criteria}

In the following, the separation $\Delta P$ will be equal to the $\mathrm{ZOI}$ size $\ell$ in order to use only independent measurement data. It is proposed to characterize a pattern by three different quantities. Associated to some of these parameters, criteria are to be defined to guarantee a priori correlation results. The following choice is made:

- A global information on the gray level distribution of a picture is given by its histogram. This information is usually checked by the user to avoid low/high level saturation and to use most of the dynamic range of the camera. Figure 2 shows an example of a gray level distribution for an artificial texture. Its histogram corresponds to a non-uniform distribution of gray levels. No significant saturation is observed and most of the dynamic range of the 8-bit camera is used.

- The previous representation is global (i.e., associated with the whole picture). A more local quantity relevant to each ZOI is the standard deviation $\sigma_{\text {ZOI }}$ of its gray level distribution $f_{\text {ZOI }}$. As noted before, a correlation algorithm does not work with a uniform gray level distribution. Consequently, a first criterion is related to a minimum value $\sigma_{\min }$ of $\sigma_{\mathrm{ZOI}}$, or similarly to a discrimination threshold (see Equation (9)).

- The autocorrelation function of each analyzed ZOI of the picture characterizes its two-point distribution. A representative "speckle" size (i.e., correlation radius) for each $\mathrm{ZOI}$ is sought. In a discrete setting, the autocorrelation function is defined as

$$
A_{\mathrm{ZOI}}(I, J)=f_{\mathrm{ZOI}} \star f_{\mathrm{ZOI}}(I, J)=\sum_{i=1}^{2^{n}} \sum_{j=1}^{2^{n}} \tilde{f}_{\mathrm{ZOI}}(i, j) \tilde{f}_{\mathrm{ZOI}}(i+I, j+J)
$$


where $\tilde{f}$ is the periodic representation of the gray level distribution $f$ of each ZOI. Figure 3 shows the autocorrelation function of a given 32-pixel ZOI. The DC component of the ZOI has been removed, and the autocorrelation function has been normalized by its maximum value reached when $I=0$ and $J=0$. The (normalized) autocorrelation function $\hat{A}_{\mathrm{ZOI}}$ is interpolated as

$$
\hat{A}_{\mathrm{ZOI}}(x, y) \approx 1-\left(a x^{2}+2 b x y+c y^{2}\right)
$$

where $a, b, c$ are identified by considering the 8 nearest points to the origin, $x$ and $y$ are the continuous coordinates around the maximum point located at the origin. Two correlation radii $R_{1}$ and $R_{2}$ are corresponding to the principal axes of the ellipse defined as the intersection of the interpolated autocorrelation function with the zero height plane

$$
\left\{\begin{array}{l}
R_{1}^{-2}=\frac{a+c}{2}+\sqrt{\left(\frac{a-c}{2}\right)^{2}+b^{2}} \\
R_{2}^{-2}=\frac{a+c}{2}-\sqrt{\left(\frac{a-c}{2}\right)^{2}+b^{2}}
\end{array}\right.
$$

The ratio between the two principal radii is a measure of the texture anisotropy [19] and the half-sum $R$ is used to evaluate an average correlation radius of any considered ZOI. A second criterion is related to the comparison of the correlation radius $R$ and the ZOI size. A value $\rho=R / \ell$ corresponds to a fraction of the ZOI size and a maximum value $\rho_{\max }$ is introduced.

The limit values of the last two criteria (i.e., $\sigma_{\min }$ and $\rho_{\max }$ ) are determined in the following analysis. 


\subsection{Choice and validation of critical values}

As discussed in Section 2, digital image correlation cannot be used with a homogeneous distribution of gray levels. Let us still consider a uniform gray level distribution, i.e., all gray levels have the same probability to be present in the ZOI, the standard deviation in this case is equal to

$$
\sigma_{\mathrm{ZOI}}=\frac{\Delta N_{\mathrm{ZOI}}}{\sqrt{12}}
$$

where $\Delta N_{\text {ZOI }}$ denotes the dynamic range of a ZOI. In many applications, a 0.05-pixel discrimination threshold is acceptable. Consequently, for each ZOI of the studied picture, the gray level standard deviation must be greater than 6 gray levels (see Equation (9))

$$
\sigma_{\min }=6 \text { gray levels }
$$

For more severe applications, this value has to be increased, and also when greater than 8-bit dynamic ranges are used.

To apply digital image correlation to a given window, it is preferable that at least one speckle (i.e., a black or white dot) and its neighborhood (i.e., a white or black zone) belong to any ZOI. Consequently, for each ZOI, the ratio between the correlation radius related to the ZOI size must not be greater than 0.25

$$
\rho_{\max }=0.25
$$

The following points can be underlined. First, this type of approach allows one to choose a ZOI size that is the most fundamental parameter of a DIC 
technique. Each new picture is analyzed independently of previous results. If a natural texture is considered, the user then knows whether it is acceptable in terms of "quality" for a DIC algorithm. Conversely, when an artificial pattern is created, the user has a way of characterizing its overall properties. If the result is not satisfactory, the pattern may be recreated again. Second, it is an a priori analysis. Third, there is a need to relate the critical values associated with the texture to the performance of a DIC code in terms of displacement and strain uncertainties associated to a spatial resolution.

\subsection{Performance evaluation and texture analysis}

The aim of this study is to evaluate the standard displacement and strain uncertainties, i.e., "the parameter that characterizes the dispersion of the quantity values that are being attributed to a measurand, based on the information used. The parameter may be, for example, a standard deviation called standard measurement uncertainty" [17]. In practice, the discrimination threshold is less than the standard displacement uncertainty, thus the latter will be assessed. Furthermore, the actual value of the prescribed displacement is not always easy to evaluate. However, fluctuations can be measured and therefore the standard measurement uncertainty is one key quantity to assess the overall performance associated to a given picture.

\subsubsection{Description of a test case}

The texture analysis is related to a picture taken from a tensile test on a single fiber with an aluminum coating itself embedded in an epoxy resin [23]. A 50$\mathrm{mm}$ lens allows one to monitor a $13 \times 9 \mathrm{~mm}$ surface (Figure 4) where a black 
and white artificial texture was created. The picture was taken by an 8-bit CCD camera whose resolution is $768 \times 576$ pixels. The correlation parameters are chosen by following the previous a priori texture analysis. Four different steps are performed as illustrated in Figure4:

- a rigid body motion is artificially applied to this picture. Ten images are created with displacement increments of 0.1 pixel. Two different algorithms are used. The first one is a pixel by pixel interpolation algorithm that linearly interpolates the gray level. The second one uses FFTs and the shift/modulation property. No significant differences between the two methods are observed;

- the eleven-image sequence is analyzed with a DIC software to evaluate the displacements for each image (here CORRELI ${ }^{\mathrm{LMT}}[5]$ );

- Once all pictures have been analyzed, the mean displacement error and the corresponding standard uncertainty are determined (Figure 5). The standard displacement uncertainty is evaluated as the mean of the standard uncertainties for the analyzed sequence. For a given ZOI size, the maximum uncertainty is reached when the prescribed displacement is equal to 0.5 pixel. In this case, the information between each ZOI in the reference and deformed pictures is the most biased. It is worth noting that if successive pictures of the undeformed state are taken, it is virtually impossible to evaluate the maximum correlation errors since they are not achieved for very small displacements but for 0.5 pixel;

- For strains, the same eleven pictures are used. It is expected that no strains should be observed since only rigid body motions are prescribed. The same procedure as for displacements is performed and the mean error and the standard strain uncertainty are calculated from strain fields as shown in Figure 5. By using this procedure, the derivation algorithm to get the strain 
field is tested too, here the one proposed by Geers et al. [22]. The use of another derivation scheme and another spatial resolution would change the strain performance. In terms of uncertainty, the same overall tendency as for displacements is observed. In particular the maximum value is reached when the prescribed displacement is equal to 0.5 pixel. These results suggest that a fast uncertainty evaluation only needs one uncertainty estimate for a 0.5-pixel displacement and the corresponding average is approximately equal to half of the latter.

It has to be noted that even if this procedure uses real pictures, all "experimental" noise that exists during an experiment is avoided (e.g.,lighting, vibrations, texture variations due to strain). If this uncertainty study is applied for different ZOI sizes, it is possible to compare the different performances. In the present case, the texture analysis (Figure4) of the picture shown in Figure 2 indicates that a 64-pixel ZOI is the appropriate interrogation window size for the DIC procedure used herein. According to the uncertainty study, for all ZOI sizes reported in Figure 6, the standard displacement uncertainty for the optimized parameter is about of $3.6 \times 10^{-3}$ pixel and the standard strain uncertainty is of the order of $5 \times 10^{-5}$. Performance comparisons can be made between different ZOI sizes and, in this case, the optimized parameter gives two times better results than with a 32-pixel ZOI size.

Last, when studying the uncertainties versus the ZOI size, one shows that there exists a trade off between displacement (and strain) uncertainties and their associated spatial resolution. Figure 6-b shows the displacement uncertainty for different ZOI sizes. If the ZOI size increases, the spatial resolution is degraded, i.e., fewer independent measurement points are obtained. This trend can be described by a power law fit relating the displacement uncertainty $\sigma_{U}$ 
to the ZOI size $\ell$

$$
\sigma_{U}=\frac{A^{\alpha+1}}{\ell^{\alpha}}
$$

where $A$ is a constant equal to 1.1 pixels in the present case (see Figure $6-b$ ) and $\alpha=1.3$ the corresponding power. The same trend is observed for strains. In the present case, the separation $\Delta P$ is equal to the $\mathrm{ZOI}$ size $\ell=2^{n}$ pixels. Figure 6-d shows the change in the mean strain uncertainties with the ZOI size when the displacement range varies between 0 and 1 pixel. A power law with an exponent $\beta$ of the order of 2.2 is obtained for the mean strain uncertainty $\sigma_{\epsilon}$ as a function of $\ell$

$$
\sigma_{\epsilon}=\frac{B^{\beta}}{2 \ell^{\beta}}
$$

with $B=0.9$ pixel. This last result shows that the two uncertainties can be related by (see Equation (10) for a similar result in terms of discrimination threshold)

$$
\sigma_{\epsilon}=C \frac{\sigma_{U}}{2 \Delta P}
$$

since $\alpha+1 \approx \beta$. In the present case, the constant $C$ is of the order of 0.6 (i.e., $C A^{\alpha+1} \approx B^{\beta}$ ).

\subsubsection{Statistical study}

The previous analysis is performed on a set of images obtained when analyzing a mechanical test. A statistical study is carried out by using 31 different 8-bit textures. Among these 31 images, 27 are artificial textures observed with classical lenses, one is a natural texture observed with classical lens, one 
is an artificial texture observed with a far-field microscope, two are natural textures observed with a far-field microscope. The correlation parameters are determined by using the critical values chosen before. Standard strain and displacement uncertainties are evaluated and a histogram is plotted in Figure 7.

¿From the standard displacement uncertainty histogram, it is concluded that the mean value is about $1.2 \times 10^{-2}$ pixel and the associated standard deviation is about $4.9 \times 10^{-3}$ pixel. In the present case, a Gaussian fit is reasonable as shown in a normal probability plot in Figure 7. From the standard strain uncertainty histogram, the mean value is ca. $2.6 \times 10^{-4}$ and the associated standard deviation is about $1.6 \times 10^{-4}$. Again, a Gaussian description seems reasonable. Among the four last textures, one gives better results than the average, two slightly worse and the last one exactly the average value. Even if these four images represent only $13 \%$ of the population, their results are not different from the overall population. Consequently, the type of texture (i.e., artificial or natural) and observation scale (i.e., classical lens vs. far-field microscope) are second order parameters compared with the property of the textures studied herein.

When using the texture criteria proposed herein, three cases occur, either the gray level standard deviation (i.e., two cases among the 31 studied) is the limiting factor, or the correlation radius ratio (i.e., 17 out of 31 ) is the only limitation, or both of them are limits (i.e., 12 out of 31). Table 1 shows a comparison between standard uncertainties and mean errors for each group quoted above and relates them to average values. Concerning the performance of each of the three groups compared to the average for both strain and displacement standard uncertainties, the first one has a better performance, the second one a slightly better one too, and the third one a slightly worse performance. The 
choice of the ZOI size given by the texture analysis yields sufficiently good results for most applications. It can be considered that by using this procedure the average performances described above can be reached.

\section{Tensile test on an aluminum alloy with strain gauges}

In this section, the critical values are tested when analyzing a tensile test performed on a 2024 aluminum alloy specimen. Strains deduced from displacement measurements are compared with strain gauge data. A special setup is used to avoid spurious flexure [24]. A far-field microscope monitors a $4 \mathrm{~mm}^{2}$ surface (Figure 3). The measurements obtained by digital image correlation are shown to be sufficient to determine the elastic properties of the alloy with a conventional analysis or with the so-called constitutive equation error technique [25].

¿From the reference image, a texture study is performed to choose the ZOI size with the proposed texture criteria. The results are shown in Figure 8. The gray level histogram shows a good distribution without any saturation. A large part of the gray level range is used. The gray level standard deviation criterion validates the 32 -pixel size but the correlation radius requires the ZOI size to be augmented to 64 pixels. As for the a priori uncertainty study, the separation $\Delta P$ is equal to the ZOI size so that each measurement "point" is completely independent of the other ones. Strains are again computed by using the procedure proposed by Geers et al. [22].

Let us compare the gauges and the digital image correlation results when the ZOI size recommended by the texture analysis (i.e., 64 pixels) is used. In the present case, the region of interest for the correlation calculation is identical 
to the gauge size so that a direct comparison can be made. The mean strain error, representing the difference between digital image correlation results for a 64-pixel ZOI size and the gauge data (longitudinal and transverse strains), is less than $5 \times 10^{-5}$. In Figure 10, different ZOI sizes are tested and the corresponding mean strain errors are evaluated when all the 21 load levels are considered. Except for ZOI size of 8 pixels, mean strain errors are less than $10^{-4}$

The results in terms of experimentally observed standard strain uncertainty (i.e., $3.4 \times 10^{-4}$, see Table 2) may appear disappointing in comparison with the average value obtained with the previous texture analyses (i.e., $2.6 \times$ $10^{-4}$ Table 1) since a $30 \%$ difference is found. However, the correct order of magnitude is found and this is directly related to the fact that the present procedure does not account for all experimental uncertainties (e.g., associated with the image acquisition process on top of the correlation algorithm itself). Even if the actual performances are (slightly) underestimated, the texture analysis is a simple tool to evaluate a priori and very quickly the texture properties.

One question remains, is the a priori numerical analysis an adapted tool for evaluating the performance of a correlation algorithm prior to performing an experiment? Figure 10 shows the answer. The experimentally determined and a priori evaluated standard strain uncertainties for each ZOI size in both longitudinal and transverse directions are compared. In a log-log plot, a very good power law fit is obtained with an exponent of -1.5 for any strain under scrutiny. There is only a multiplicative offset by a factor of the order of 1.3. It is therefore concluded that the a priori uncertainty study is validated and is therefore an appropriate way of evaluating the overall performance of 
a DIC algorithm. Furthermore, the a priori uncertainty study is particularly adapted to help the user choose the correlation parameters. For this procedure, which only needs one single (reference) image shifted by an amount of 0.5 pixel, the user evaluates the displacement and strain errors and standard uncertainties. The texture analysis, which is faster, allows one to choose correlation parameters associated to average performances. It is likely to be easier to use for unexperienced users. More experienced users will want to know more precisely, in each particular case, the standard measurement uncertainties.

\section{Conclusion}

A texture analysis has been developed and tested in this paper to choose correlation parameters of a DIC code. Two criteria are proposed and are related to the standard deviation of the gray level distribution in each interrogation window, and a representative "speckle" size (i.e., correlation radius). When critical parameters are chosen (e.g., a minimum standard deviation of 6 gray levels, and a maximum correlation radius equal to one quarter of the ZOI

size), an average value of standard displacement uncertainty of $1.2 \times 10^{-2}$ pixel is obtained for 31 different textures. The corresponding average value of the standard strain uncertainty is equal to $1.6 \times 10^{-4}$.

If the user wants a more accurate estimate of the performances, a displacement is artificially prescribed to a picture. Steps of 0.1 pixel are applied from 0 to 1 pixel. The standard displacement and strain uncertainties are calculated. The overall performance is then given by the mean value of the two uncertainty functions. It can be noted that with the present correlation algorithm, the mean uncertainties are equal to one half of the maximum value corresponding 
to a 0.5-pixel displacement. This result is obtained systematically so that a practical way only consists in considering only one displacement level (i.e., 0.5 pixel) to get the sought result. With this simplified approach, the computation time is reduced by one order of magnitude.

The texture analysis has been tested when analyzing a tensile test on an aluminum alloy where digital image correlation results have been compared to strain gauge measurements. With the two above criteria, the proposed choice of correlation parameters gives good results and validates the present approach. Furthermore, the present analysis also shows that the a priori estimates of standard strain uncertainties are in good agreement with values obtained in the analyzed experiment when considering the strain gauge results as reference data. It is therefore recommended to use this last approach that quantifies a priori the performance of a correlation algorithm for different correlation parameters, for example the ones obtained from the texture analysis.

\section{Acknowledgments}

This work was carried out as a collaboration within the CNRS research network "Mesure de champs et identification en mécanique des solides."

\section{References}

[1] M.A. Sutton, S.R. McNeill, J.D. Helm and Y.J. Chao, "Advances in TwoDimensional and Three-Dimensional Computer Vision," in Photomechanics, P.K. Rastogi, eds., (Springer, Berlin (Germany), 2000), pp. 323-372. 
[2] B. Wagne, S. Roux and F. Hild, "Spectral Approach to Displacement Evaluation From Image Analysis," Eur. Phys. J. AP 17, pp. 247-252 (2002).

[3] P.J. Burt, C. Yen and X. Xu, "Local correlation measures for motion analysis: a comparative study," in Proc. IEEE Conf. on Pattern Recognition and Image Processing (1982), pp. 269-274.

[4] M.A. Sutton, W.J. Wolters, W.H. Peters, W.F. Ranson and S.R. McNeill, "Determination of Displacements Using an Improved Digital Correlation Method," Im. Vis. Comp. 1, pp. 133-139 (1983).

[5] F. Hild, B. Raka, M. Baudequin, S. Roux and F. Cantelaube, "Multi-Scale Displacement Field Measurements of Compressed Mineral Wool Samples by Digital Image Correlation,” Appl. Optics IP 41, pp. 6815-6828 (2002).

[6] P. Forquin, L. Rota, Y. Charles and F. Hild, "A Method to Determine the Toughness Scatter of Brittle Materials," Int. J. Fract. 125, pp. 171-187 (2004).

[7] D.S. Dawicke and M.S. Sutton, "CTOA and Crack-Tunneling Measurements in Thin Sheet 2024-T3 Aluminum Alloy," Exp. Mech. 34, pp. 357-368 (1994).

[8] M.A. Sutton, S.R. McNeill, J. Jang and M. Babai, "Effects of subpixel image restoration on digital correlation error estimates," Opt. Eng. 27, pp. 870-877 (1988).

[9] F. Hild, J.-N. Périé and M. Coret, Mesure de champs de déplacements 2D par intercorrélation d'images : CORRELI 2D , report 230, LMT-Cachan, (1999).

[10] D. Jeulin and P. Laurenge, "Simulation of Rough Surfaces by Morphological Random Functions," J. Electr. Imaging 6, pp. 16-30 (1997).

[11] L.I. Goldfischer, "Autocorrelation function and power spectral density of laserproduced specke patterns," J. Opt. Soc. Am. A 55, pp. 247-253 (1965).

[12] S. Lowenthal and H. Arsenault, "Image formation for coherent diffuse objects: statistical properties," J. Opt. Soc. Am. 60, pp. 1478-1483 (1970). 
[13] T.L. Alexander, J.E. Harvey and A.R. Weeks, "Average speckle size as a function of intensity threshold level: comparison of experimental measurements with theory," Appl. Optics 33, pp. 8240-8250 (1994).

[14] L. Leushacke and M. Kirchner, "Three-dimensional correlation coefficient of speckle intensity for rectangular and circular apertures," J. Opt. Soc. Am. 11, pp. 671-673 (1994).

[15] P. Jacquot, "Figures de speckle : rôle en mécanique expérimentale," in Identification du comportement des matériaux et de la rupture des structures à l'aide de méthodes optiques, IPSI report, Paris, 2003.

[16] J.N. Périé, S. Calloch, C. Cluzel and F. Hild, "Analysis of a Multiaxial Test on a C/C Composite by Using Digital Image Correlation and a Damage Model," Exp. Mech. 42, pp. 318-328 (2002).

[17] ISO, International Vocabulary of Basic and General Terms in Metrology (VIM), (International Organization for Standardization, Geneva (Switzerland), 1993).

[18] J.W. Cooley and J.W. Tuckey, "An Algorithm for the Machine Calculation of Complex Fourier Series," Math. Comp. 19, pp. 297-301 (1965).

[19] S. Bergonnier, F. Hild and S. Roux, Local anisotropy analysis for non-smooth images, Patt. Recogn. 40 [2] (2007) 544-556.

[20] G. Holst, CCD Arrays, Cameras and Displays, (SPIE Engineering Press, Washington DC (USA), 1998).

[21] L. Allais, M. Bornert, T. Bretheau and D. Caldemaison, Experimental characterization of the local strain field in a heterogeneous elastoplastic material, Acta Met. Mat. 42, pp. 3865-3880 (1994).

[22] M.G.D. Geers, R. De Borst and W.A.M. Brekelmans, "Computing Strain Fields from Discrete Displacement Fields in 2D-Solids," Int. J. Solids Struct. 33, pp. 4293-4307 (1996). 
[23] K. Triconnet, K. Derrien, D. Baptiste, Y. Surrel and J. Molimard, "Local Fields Measurements: Interferometric Moiré and Digital Image Correlation," in Proc. Society of Experimental Mechanics Conf. X, (SEM, CD-ROM, 2004), 9 p.

[24] F. Hild, E. Amar and D. Marquis, "Stress Heterogeneity Effect on the Strength of Silicon Nitride," J. Am. Ceram. Soc. 75, pp. 700-702 (1992).

[25] G. Geymonat, F. Hild and S. Pagano, "Identification of elastic parameters by displacement field measurement," C. R. Mécanique 330, pp. 403-408 (2002). 


\section{List of Tables}

1 Mean values $\bar{\bullet}$ and standard deviations $\overline{\boldsymbol{\bullet}}$ of the standard displacement $\sigma_{u}$ and strain $\sigma_{\epsilon}$ uncertainties related to the criterion(a) that is(are) satisfied to choose the correlation parameters for the 31 analyzed pictures.

2 Standard strain uncertainties $\sigma_{\epsilon}$ for 3 different ZOI sizes determined with the a priori analysis (APA) and compared with actual values (Exp.) for the analyzed experiment. When using the two texture criteria, the first ZOI size to satisfy them both is 64 pixels. 
Table 1

Mean values $\boldsymbol{\bullet}$ and standard deviations $\overline{\boldsymbol{}}$ of the standard displacement $\sigma_{u}$ and strain $\sigma_{\epsilon}$ uncertainties related to the criterion(a) that is(are) satisfied to choose the correlation parameters for the 31 analyzed pictures.

\begin{tabular}{|c|c|c|c|c|c|}
\hline Criteria & Nb. of & $\bar{\sigma}_{u}$ & $\overline{\bar{\sigma}}_{u}$ & $\bar{\sigma}_{\epsilon}$ & $\overline{\bar{\sigma}}_{\epsilon}$ \\
\hline cases & $($ pixel $)$ & $($ pixel $)$ & & \\
\hline$\sigma_{\text {ZOI }}$ & 2 & $7.6 \times 10^{-3}$ & $5.5 \times 10^{-3}$ & $1.0 \times 10^{-4}$ & $7.6 \times 10^{-5}$ \\
\hline$\rho$ & 17 & $1.0 \times 10^{-2}$ & $4.3 \times 10^{-3}$ & $2.4 \times 10^{-4}$ & $1.4 \times 10^{-4}$ \\
\hline$\sigma_{\text {ZOI }} \& \rho$ & 12 & $1.5 \times 10^{-2}$ & $4.4 \times 10^{-3}$ & $3.3 \times 10^{-4}$ & $1.8 \times 10^{-4}$ \\
\hline Mean value & - & $1.2 \times 10^{-2}$ & $4.9 \times 10^{-3}$ & $2.6 \times 10^{-4}$ & $1.6 \times 10^{-4}$ \\
\hline
\end{tabular}


Table 2

Standard strain uncertainties $\sigma_{\epsilon}$ for 3 different ZOI sizes determined with the a priori analysis (APA) and compared with actual values (Exp.) for the analyzed experiment. When using the two texture criteria, the first ZOI size to satisfy them both is 64 pixels.

\begin{tabular}{|c|c|c|}
\hline$\ell$ (pixels) & APA & Exp. \\
\hline 32 & $5.9 \times 10^{-4}$ & $9.2 \times 10^{-4}$ \\
\hline 64 & $2.5 \times 10^{-4}$ & $3.4 \times 10^{-4}$ \\
\hline 128 & $6.5 \times 10^{-5}$ & $1.1 \times 10^{-4}$ \\
\hline
\end{tabular}




\section{List of Figures}

1 Spatial resolution for strain and displacement measurements with a correlation software. In both cases, the spatial resolution is $\ell=2^{n}$ pixels for displacements. The spatial resolution for the strain computation is equal to $2 \Delta P$, e.g. $2 \times 2^{n}$ pixels (a) or $2^{n}$ pixels (b).

2 -a-Texture analysis of the considered picture. -b-Corresponding gray level histogram. -c-Maximum dimensionless correlation radius $\rho_{\max }$ vs. ZOI size $\ell$. -d-Minimum gray level standard deviation $\sigma_{\min }$ vs. ZOI size $\ell$.

3 -a-Picture of an artificial texture of an aluminum alloy surface. The square box is the analyzed ZOI. Autocorrelation function before (b) and after (c) a parabolic interpolation in the vicinity of the maximum.

4 Texture analysis and performance evaluation of a digital image correlation software.

5 Displacement(a, b) and strain(c, d) mean errors and standard uncertainties for the picture of Fig. 2 with a ZOI size of 64 pixels when artificially moved by increments of 0.1 pixel.

6 Mean errors and standard uncertainties for displacements (a, b) and strains (c, d) as functions of the ZOI size $\ell$ (picture of Fig. 2). For the standard uncertainties, the solid and dashed lines a power law fits. 
$7 \quad$ Standard uncertainty results for 31 images using the ZOI parameter determined by the texture analysis. Histograms and corresponding normal probability plot for standard displacement (a, b) and strain (c, d) uncertainties.

8 -a-Texture analysis of the considered picture. -b-Corresponding gray level histogram. -c-Maximum dimensionless correlation radius $\rho_{\max }$ vs. ZOI size $\ell$. -d-Minimum gray level standard deviation $\sigma_{\min }$ vs. ZOI size $\ell$.

9 Mean strain errors between digital image correlation evaluations and strain gauge results for different ZOI sizes.

10 Comparison between experimentally determined and a priori evaluated standard strain uncertainties for the picture of Fig. 3. The solid and dashed lines a power law fits. 


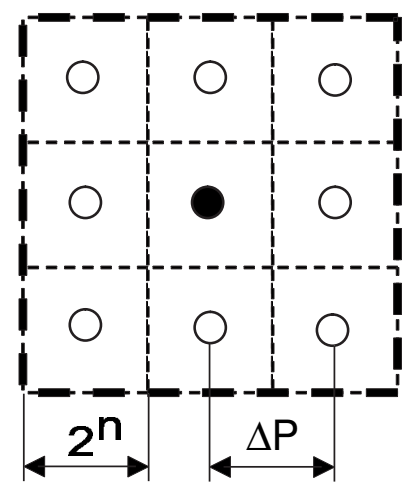

$-a-$

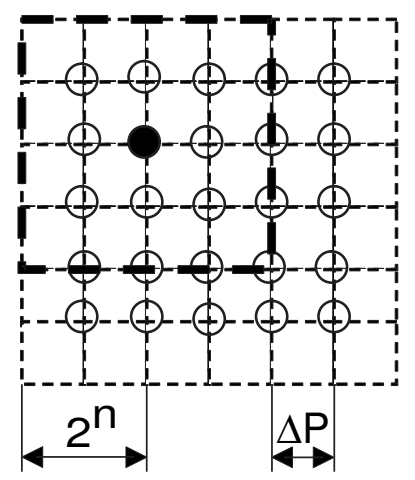

$-b-$

Fig. 1. Spatial resolution for strain and displacement measurements with a correlation software. In both cases, the spatial resolution is $\ell=2^{n}$ pixels for displacements. The spatial resolution for the strain computation is equal to $2 \Delta P$, e.g., $2 \times 2^{n}$ pixels (a) or $2^{n}$ pixels (b). 


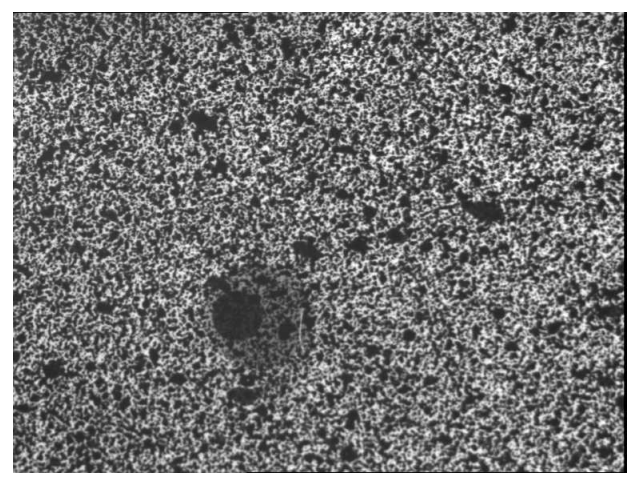

-a-

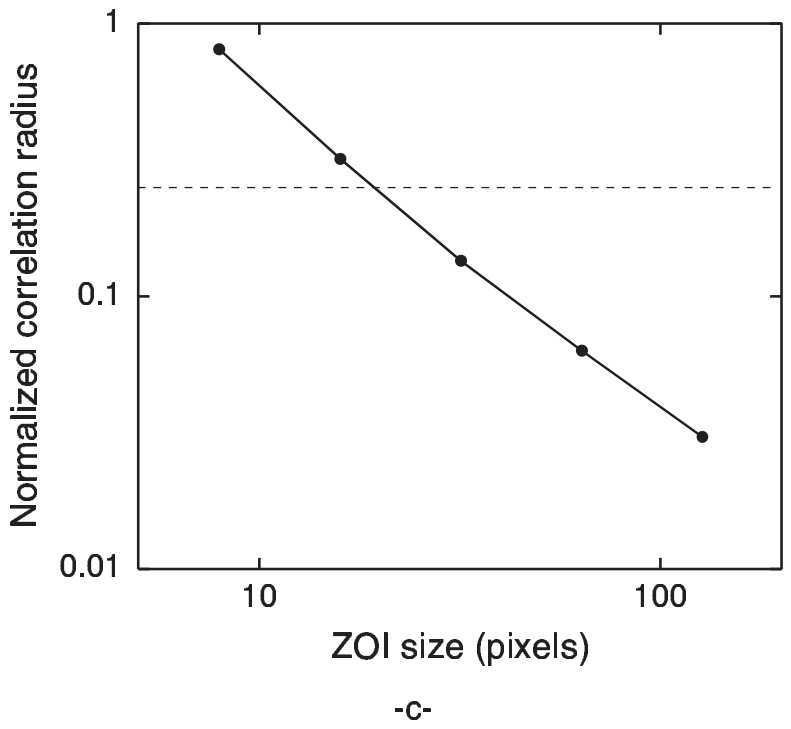

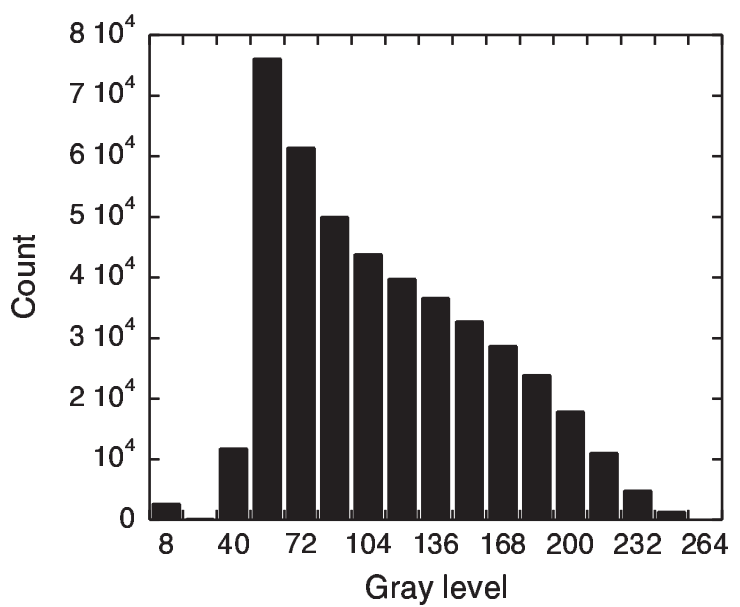

$-b-$

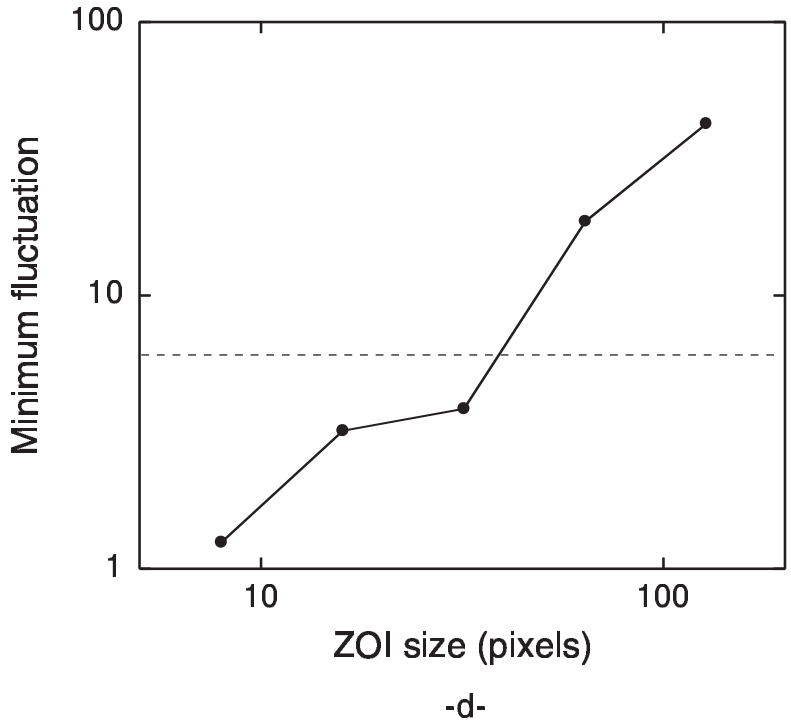

Fig. 2. -a-Texture analysis of the considered picture. -b-Corresponding gray level histogram. -c-Maximum dimensionless correlation radius $\rho_{\max }$ vs. ZOI size $\ell$. -d-Minimum gray level standard deviation $\sigma_{\min }$ vs. ZOI size $\ell$. 


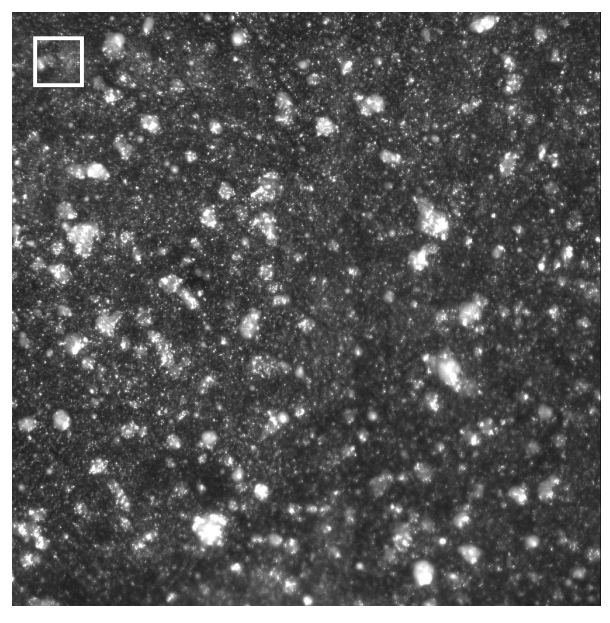

-a-

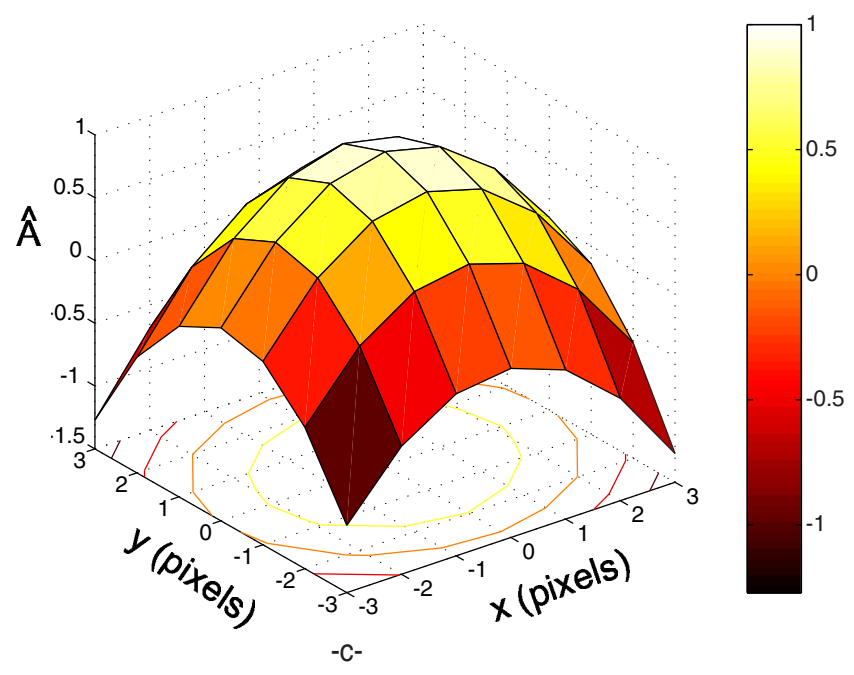

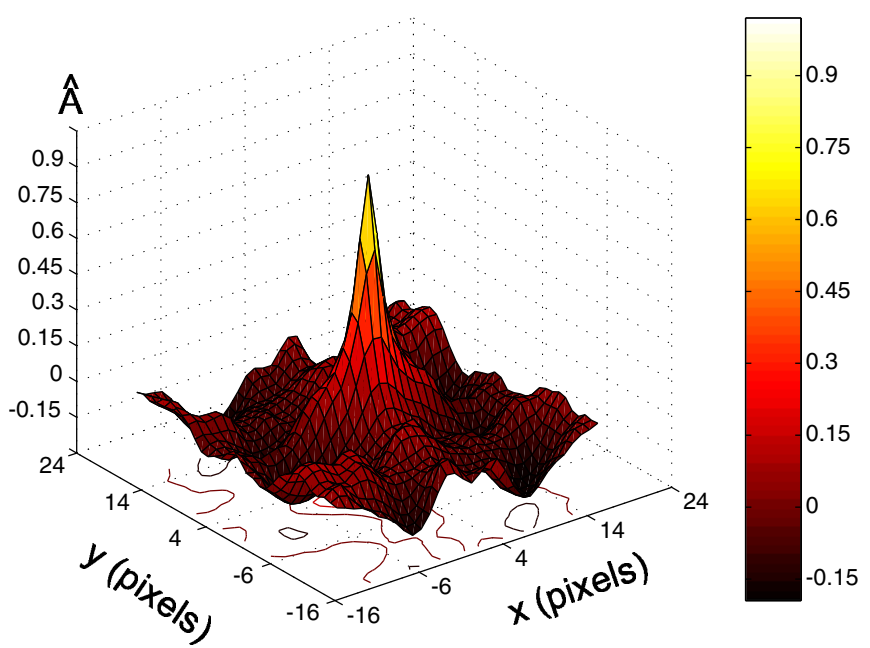

$-b-$

Fig. 3. -a-Picture of an artificial texture of an aluminum alloy surface. The square box is the analyzed ZOI. Autocorrelation function before (b) and after (c) a parabolic interpolation in the vicinity of the maximum. 


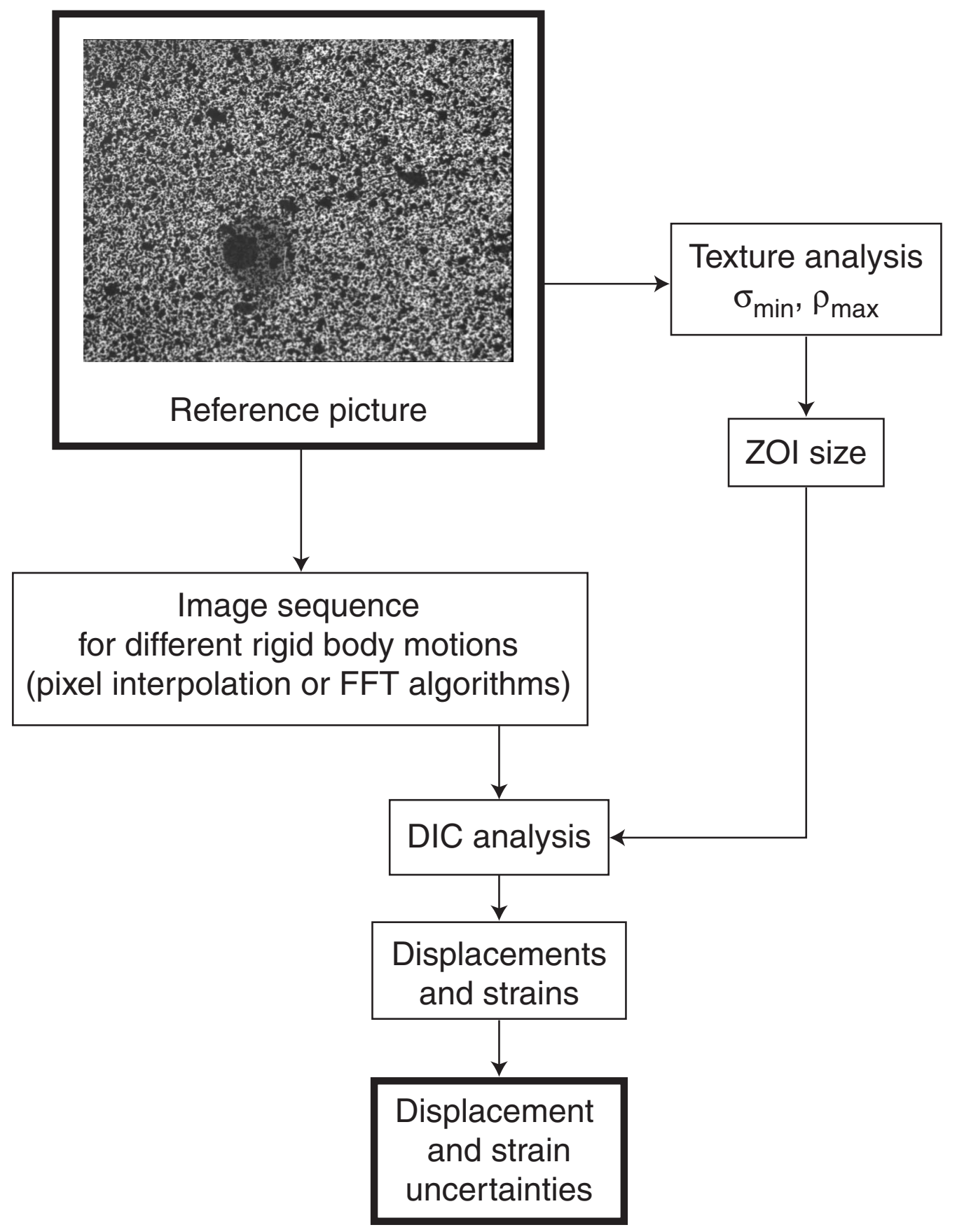

Fig. 4. Texture analysis and performance evaluation of a digital image correlation software. 


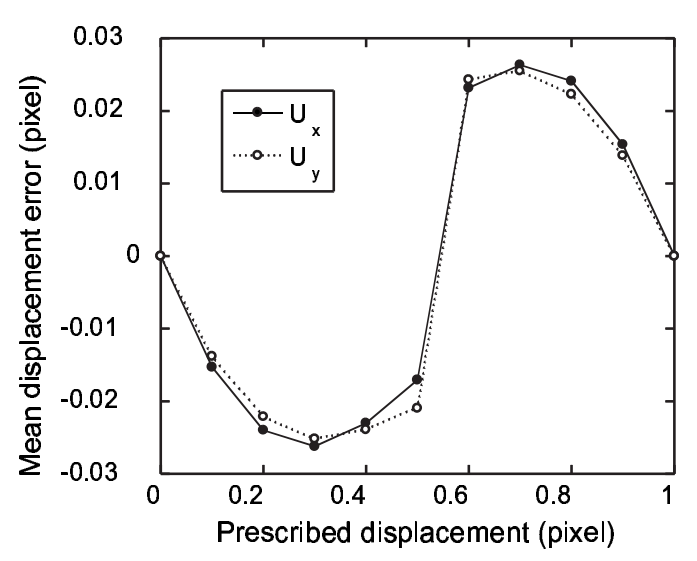

-a-

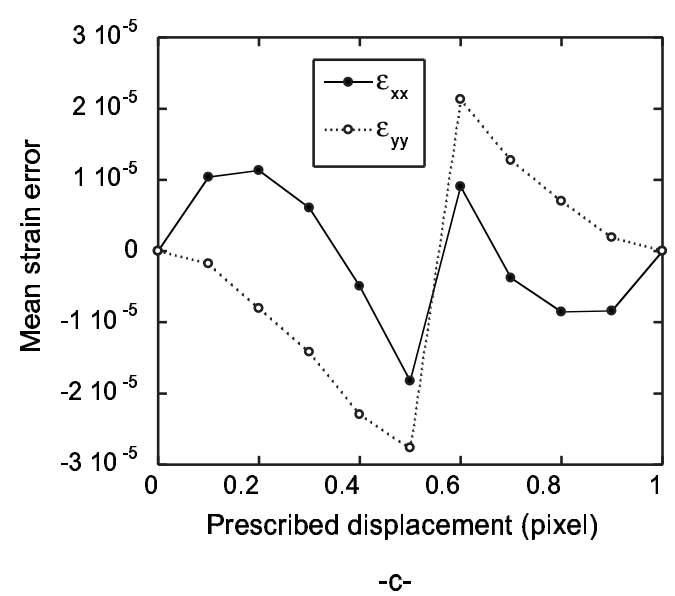

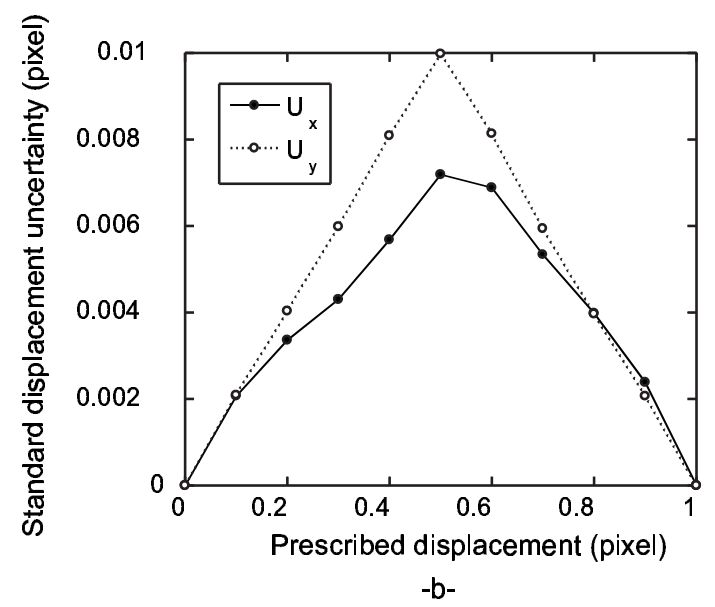

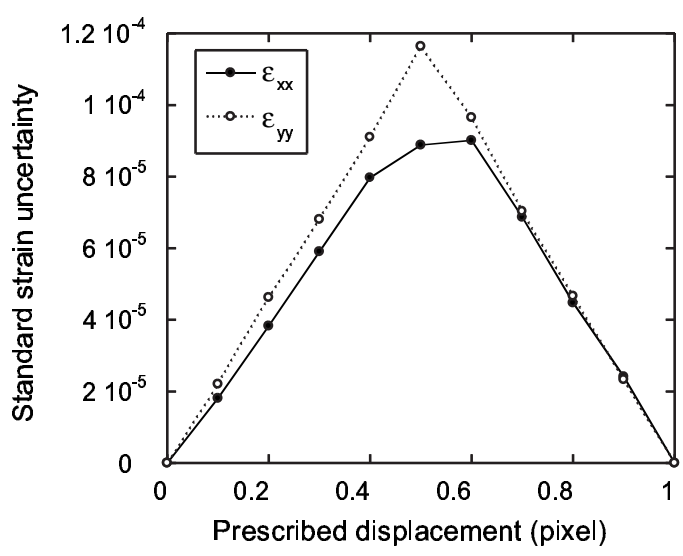

$-d-$

Fig. 5. Displacement(a, b) and strain(c, d) mean errors and standard uncertainties for the picture of Fig. 2 with a ZOI size of 64 pixels when artificially moved by increments of 0.1 pixel. 

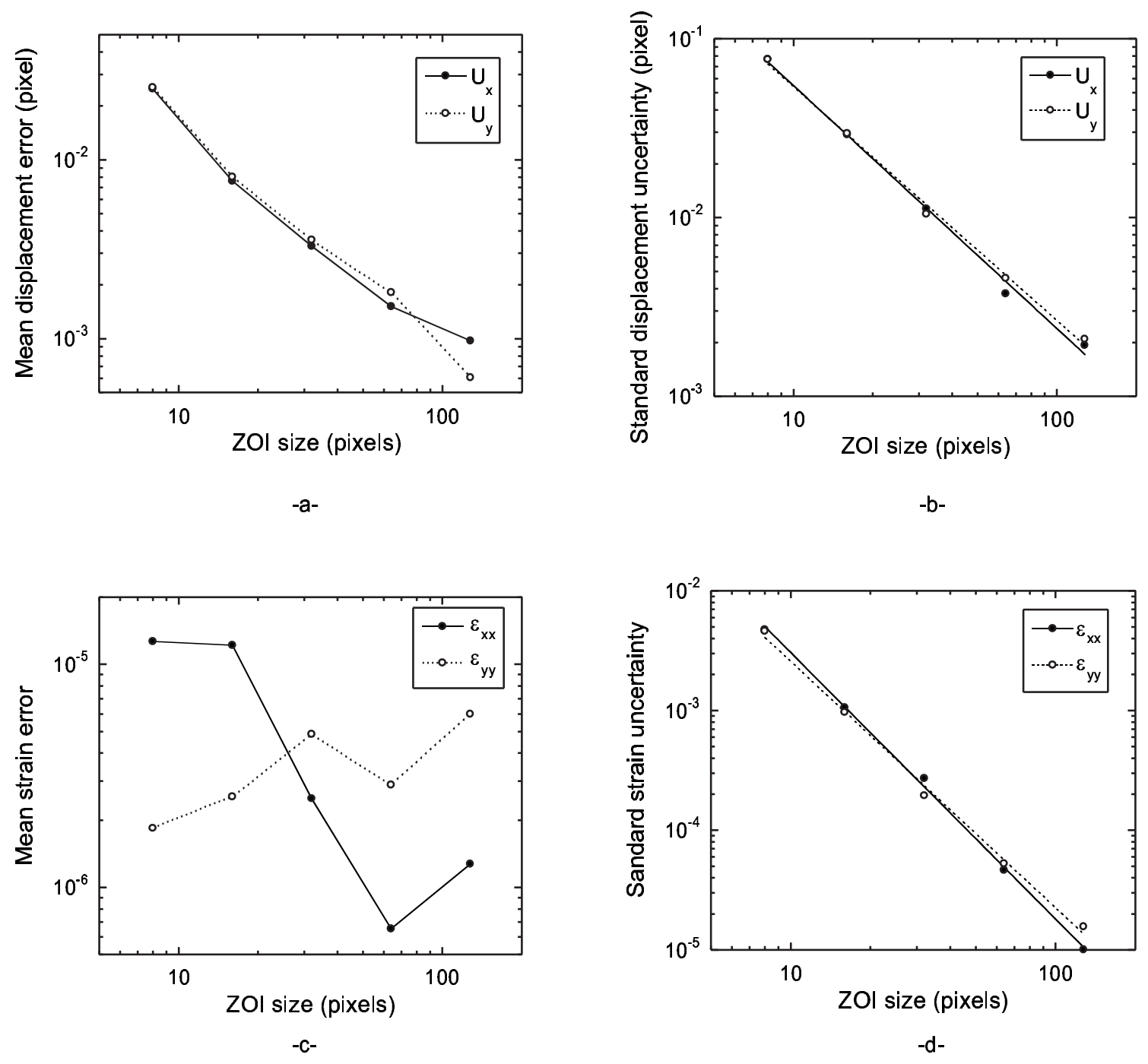

Fig. 6. Mean errors and standard uncertainties for displacements (a, b) and strains (c, d) as functions of the ZOI size $\ell$ (picture of Fig. 2). For the standard uncertainties, the solid and dashed lines a power law fits. 


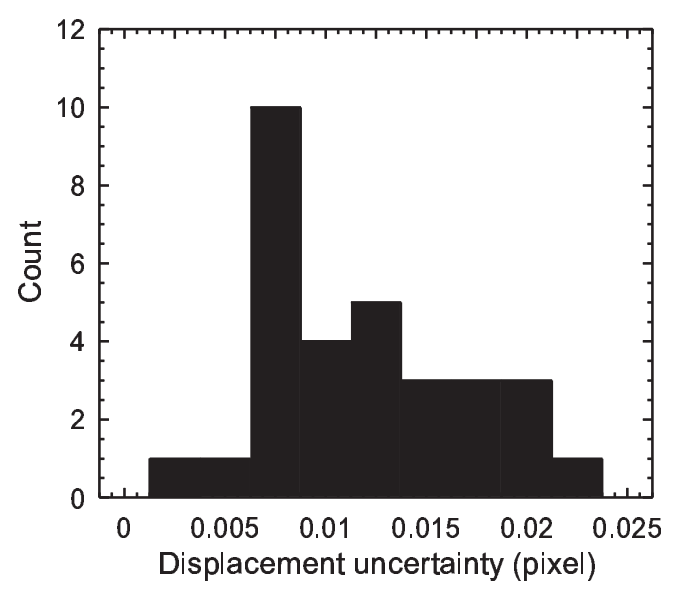

$-a-$

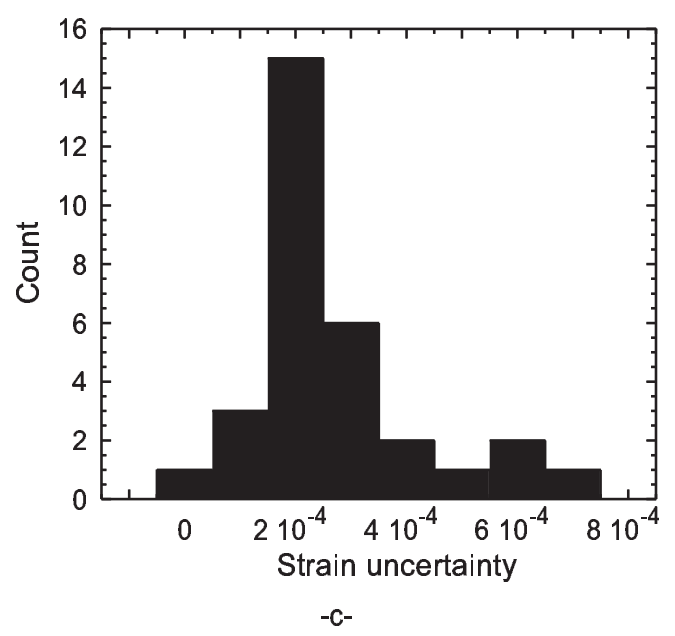

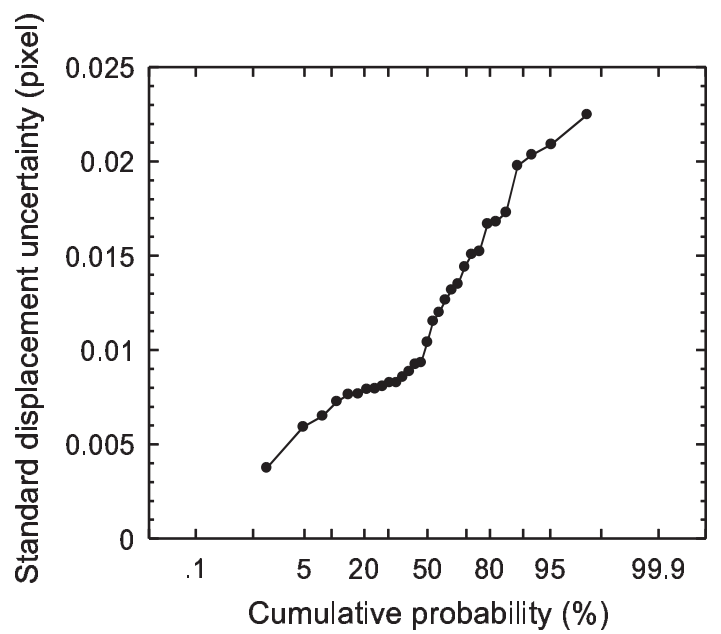

$-b-$

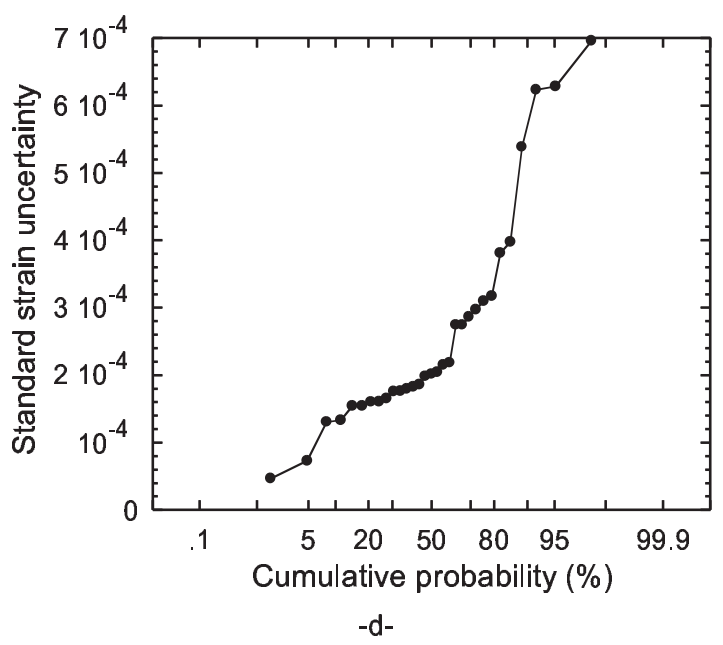

Fig. 7. Standard uncertainty results for 31 images using the ZOI parameter determined by the texture analysis. Histograms and corresponding normal probability plot for standard displacement (a, b) and strain (c, d) uncertainties. 


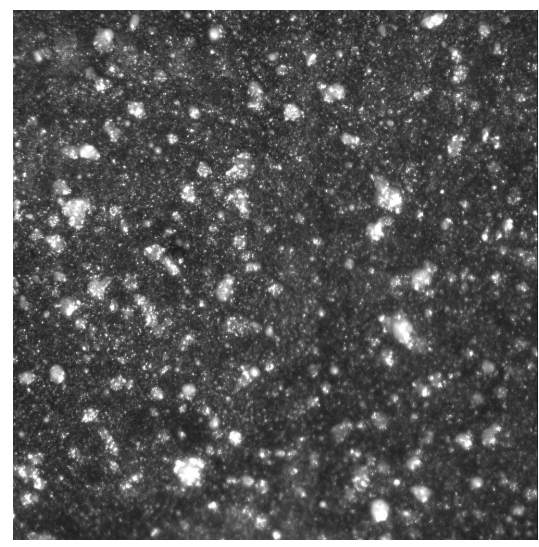

-a-

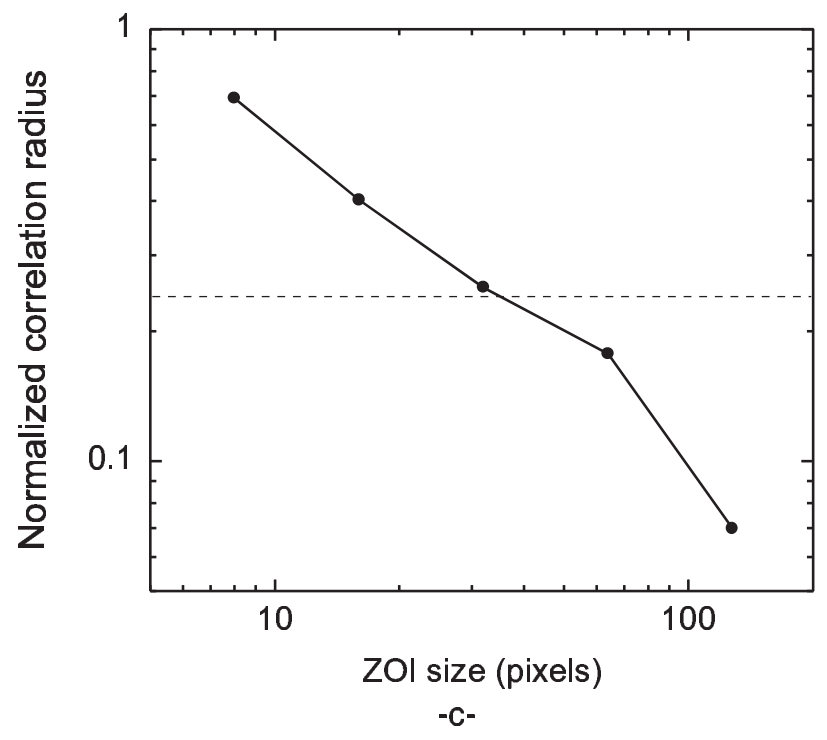

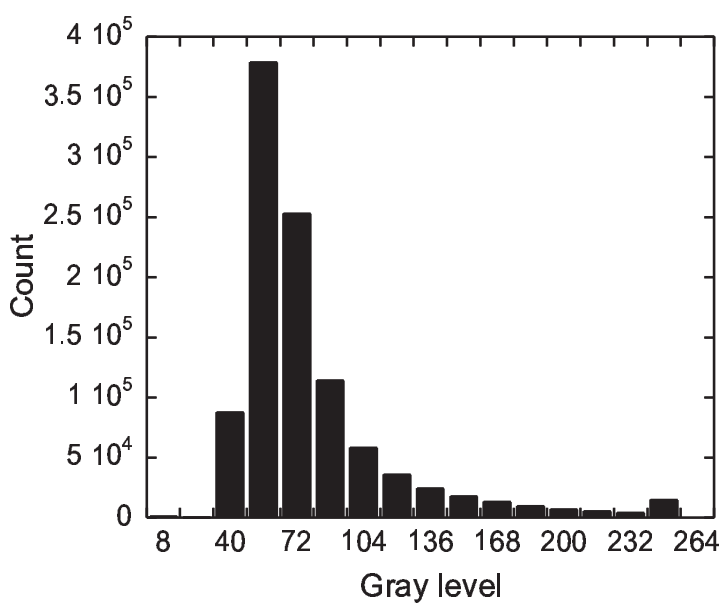

$-b-$

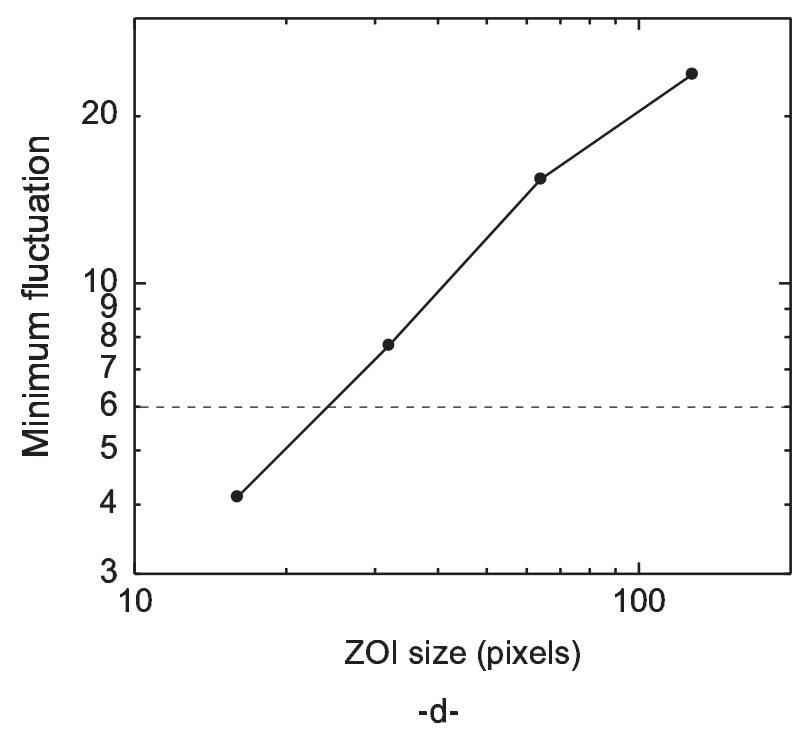

Fig. 8. -a-Texture analysis of the considered picture. -b-Corresponding gray level histogram. -c-Maximum dimensionless correlation radius $\rho_{\max }$ vs. ZOI size $\ell$. -d-Minimum gray level standard deviation $\sigma_{\min }$ vs. ZOI size $\ell$. 


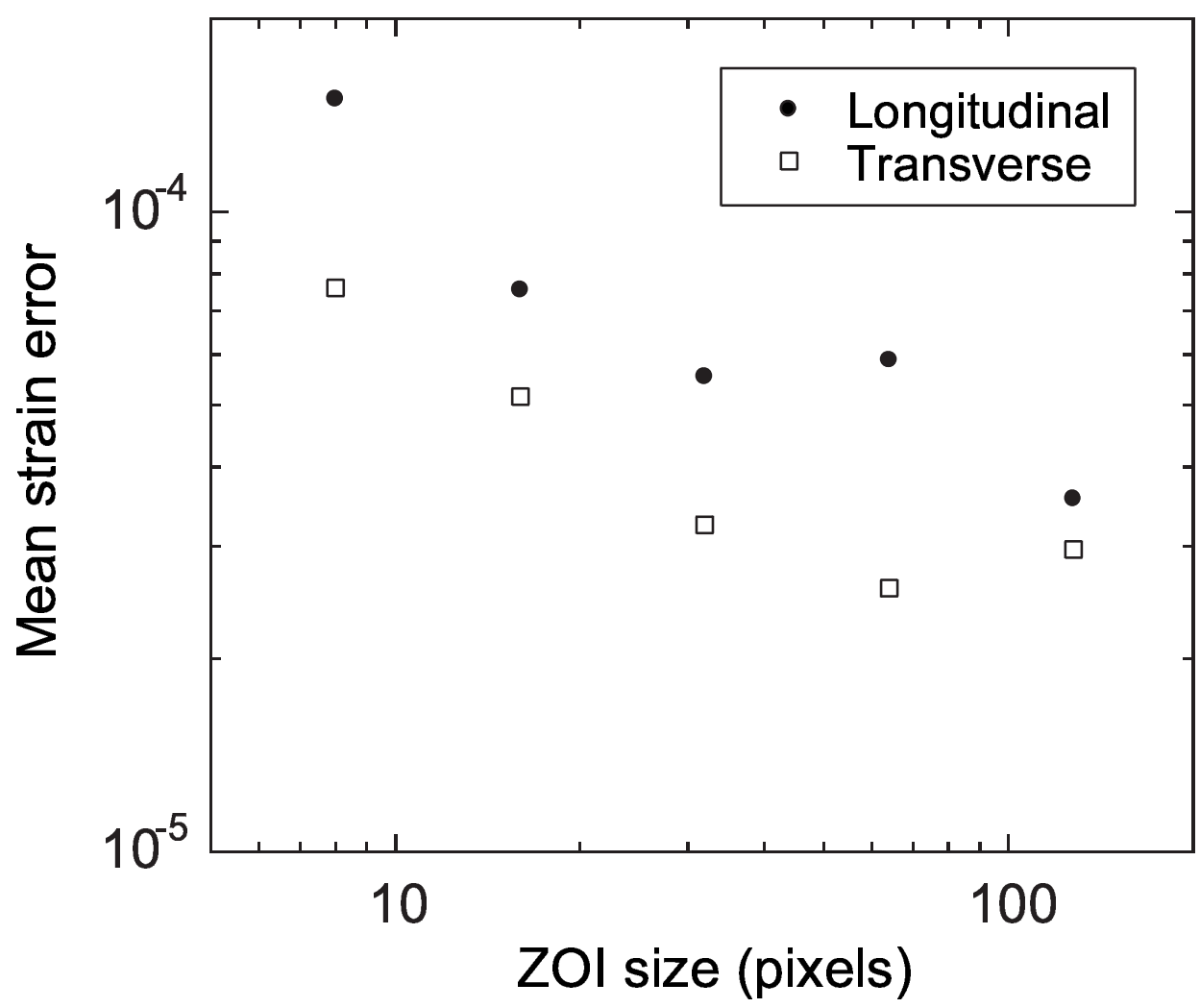

Fig. 9. Mean strain errors between digital image correlation evaluations and strain gauge results for different ZOI sizes. 


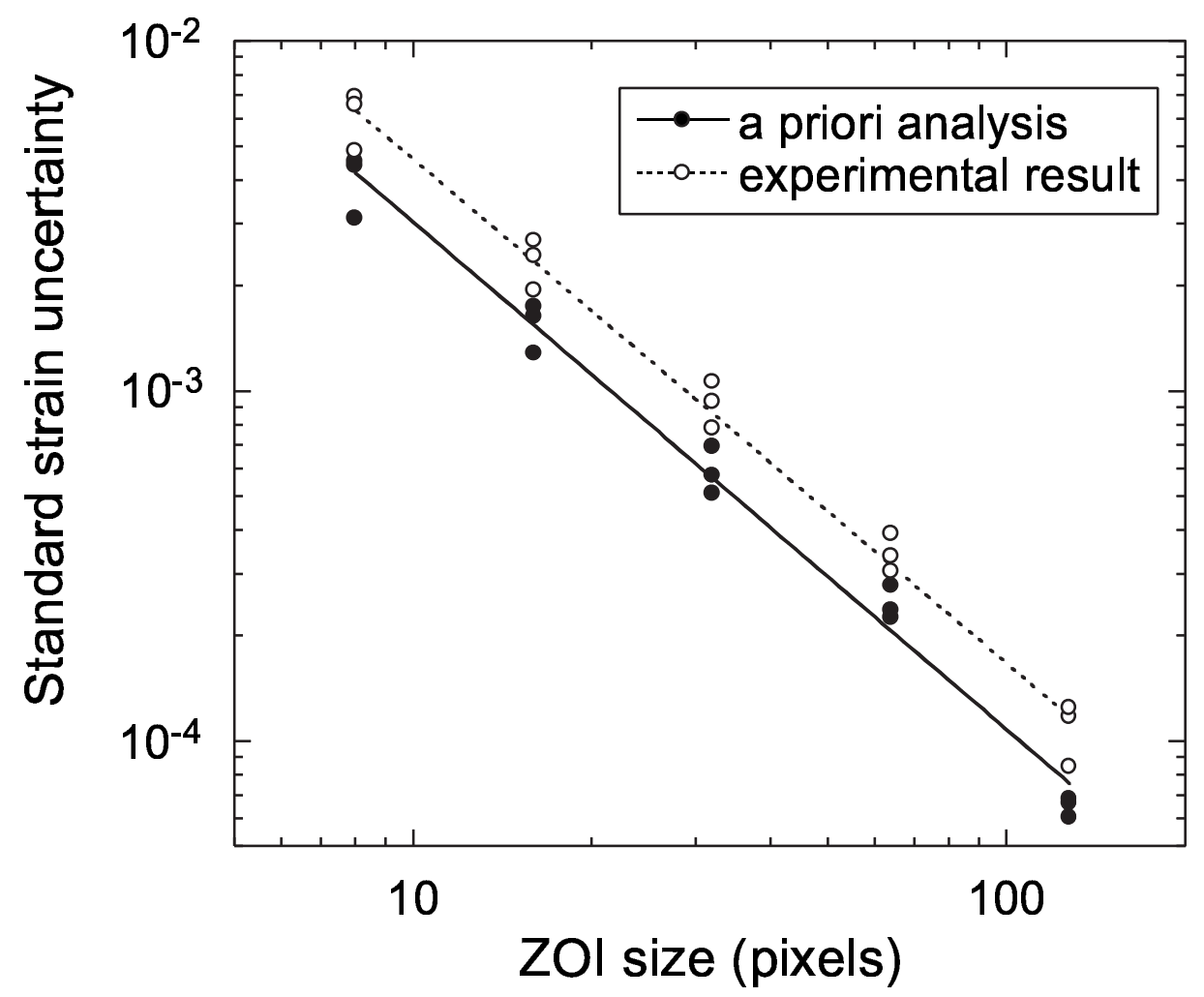

Fig. 10. Comparison between experimentally determined and a priori evaluated standard strain uncertainties for the picture of Fig. 3. The solid and dashed lines a power law fits. 